\title{
Estudio comparativo de propiedades del cemento Portland con diferentes opacificadores y MTA presentes en el mercado
}

\section{Comparative study of Portland cement properties with different opacifiers and MTA present in the market}

\section{Estudo comparativo das propriedades do cimento Portland com diferentes opacificantes e MTA presentes no mercado}

\author{
Giomara Catherine Silva iD \\ Fábio Duarte da Costa Aznar iD \\ Adriana Rodrigues de Freitas Aznar \\ Endereço para correspondência: \\ Giomara Catherine Silva \\ Faculdade do Centro Oeste Paulista - FACOP \\ Rua Luiz Gimenez Mocegose, 72 \\ Distrito Industrial \\ 17499-010 - Piratininga - São Paulo - Brasil \\ E-mail: dra.gsilva@hotmail.com
}

Recebido: 08.09.2020

Aceito: 19.09.2020

\section{RESUMEN}

Objetivo fue investigar qué clase de MTA presente en el mercado ecuatoriano y el cemento Portland con diferentes aditivos es más radiopaco. Una muestra se constituyó de 6 placas de acrílico con dimensiones iguales $(2.2 \mathrm{~cm} \mathrm{x} 4.5 \mathrm{~cm} \times 1 \mathrm{~mm})$ a una película radiográfica con seis orificios de $5 \mathrm{~mm}$ de diámetro cada uno, cada cemento fue mesclado de acuerdo con las especificaciones de cada fabricante y colocado en cada uno de los orificios: Proroot de la Densply, MTA White de la Angelus, Biodentine de la Septodont, cemento Portland sin ningún aditivo, cemento Portland con 20\% de sulfato de bario y cemento Portland con $20 \%$ de yodoformo, las placas fueron procesadas con el uso de radiovisiógrafo Fona y con un software se determinó la densidad radiográfica. Portland con yodoformo y Portland con sulfato de bario fueron los que presentaron mayor radiopacidad. Portland sin aditivos fue el que presento menor radiopacidad. En lo que se refiere a los MTA de casa comerciales Proroot presentó mayor radiopacidad y el Biodentine el que presentó menor radiopacidad. La radiopacidad es una propiedad que permite al MTA diferenciarlo en una radiografía de las estructuras limitantes, siendo Proroot el más radiopaco.

PALABRAS CLAVE: Cementos dentales. Radiografía dental. Endodoncia.

\begin{abstract}
Objective it was to investigate what kind of MTA present in the Ecuadorian market and Portland cement with different additives is more radiopaque. A sample was filtered from 6 acrylic plates with equal dimensions $(2.2 \mathrm{~cm} \mathrm{x} 4.5 \mathrm{~cm} \mathrm{x} 1 \mathrm{~mm})$ to a radiographic film with six holes of $5 \mathrm{~mm}$ diameter each, each cement was mixed according to the specifications of each manufacturer and placed in each of the holes: Proroot from Densply, MTA White from Angelus, Biodentine from Septodont, Portland cement without any additives, Portland cement with $20 \%$ barium sulfate and Portland cement with $20 \%$ of iodoform, the plates were processed using a Fona radiovisiograph and the radiographic density was determined with software. Portland with iodoform and Portland with barium sulfate were the ones with the highest radiopacity. Portland without additives was the
\end{abstract}


one with the lowest radiopacity. Regarding commercial MTAs, Proroot presented the highest radiopacity and Biodentine the one with the lowest radiopacity. Radiopacity is a property that allows MTA to differentiate it from limiting structures in an Xray, Proroot being the most radiopaque.

KEYWORDS: Dental cements. Radiography, dental. Endodontics.

\section{RESUMO}

O objetivo foi investigar que tipo de MTA presente no mercado equatoriano e o cimento Portland com diferentes aditivos é mais radiopaco. Uma amostra consistiu de 6 placas de acrílico com dimensões iguais $(2.2 \mathrm{~cm} \mathrm{x} 4.5 \mathrm{~cm} \mathrm{x} 1 \mathrm{~mm})$ a um filme radiográfico com seis orifícios de $5 \mathrm{~mm}$ de diâmetro cada, cada cimento foi misturado de acordo com as especificações de cada fabricante e colocado em cada um dos orifícios: Proroot da Densply, MTA White da Angelus, Biodentine da Septodont, cimento Portland sem quaisquer aditivos, cimento Portland com $20 \%$ de sulfato de bário e cimento Portland com $20 \%$ de iodofórmio, as placas foram processados com o uso de radiovisiógrafo Fona e com software, foi determinada a densidade radiográfica, sendo Portland com iodofórmio e Portland com sulfato de bário os que apresentaram maior radiopacidade. Portland sem aditivos foi o que apresentou menor radiopacidade. Em relação aos MTAs comerciais, o Proroot apresentou a maior radiopacidade e o Biodentine o que apresentou a menor radiopacidade. A radiopacidade é uma propriedade que permite ao MTA diferenciá-la em um raio-X das estruturas limitantes, sendo Proroot a mais radiopaca.

PALAVRAS-CHAVE: Cimentos dentários. Radiografia dentária. Endodontia. 


\section{INTRODUCCIÓN}

El MTA es un material el cual tiene como materia prima a el cemento Portland y es usado para varios de los procedimientos endodónticos ${ }^{1}$.

El Agregado Trióxido Mineral (MTA) es un material que fue descrito por primera vez en un artículo científico dental ${ }^{1}$, pero fue patentado en $1995^{2}$.

Se ha convertido en el material ideal para diversos propósitos, como el sellado de la cámara pulpar o radiculares, resorciones externas, retroobturaciones y en el recubrimiento de la pulpa dental, pues se ha comprobado sus excelentes propiedades selladoras, reparativas, biocompatibles, antibacterianas ${ }^{3}$.

Sin embargo, MTA presenta amplia superioridad como material de retroobturación en comparación a otros, pero su inconveniente es su alto costo limitando de esa forma su uso. Su biocompatibilidad ha sido estudiada y se ha realizado comparaciones con el cemento Portland teniendo similares resultados ${ }^{4}$.

En su composición el óxido de bismuto es el responsable de la radiopacidad en el MTA, pero no en el Portland, este material es clasificado como estructural o no estructural, los que son estructurales presentan cantidades de carbonatos estos son los responsables de la resistencia ${ }^{5}$.

El material ideal podría presentar suficiente radiopacidad para ser distinguido de las diferentes estructuras dentales ${ }^{3}$.

Diversos estudios se han realizado utilizando métodos digitales que determinen mediante la evaluación de grises la radiopacidad de estos materiales, utilizando software para medir la radiopacidad utilizando un software Image Tool for Windows v.3.00 (University of Texas, Texas, USA), en un área padronizada de 3000 pixels $^{6}$.

El objetivo de este trabajo fue del comprobar la semejanza entre el MTA- Angelus, Biodentine, Pro-root de la Dentisply y cemento Portland com diferentes aditivos como son el sulfato de bario y el yodoformo en ciertas características como son la radiopacidad, característica muy importante al tener en cuenta que los tratamientos endodónticos cuando se utiliza MTA es indispensable conocer si el material está colocado de forma adecuada y para un control posterior y seguimiento posoperatorio.

Los diferentes aditivos opacificadores que se utilizados en el presente estudio son usados en la práctica médica los cuales son biocompatibles. Tal es el caso del sulfato de bario usado en exámenes gastroenterológicos como medio de contraste. El yodoformo es usado en odontopediatría con un alto poder antiséptico?

El radiopacificador ideal debe ser inerte, no tóxico y libre de contaminantes La adición de agente de radiopacificación al cemento a base de silicato de calcio debería ser suficiente para permitir su visualización en la radiografía sin comprometer otras propiedades ${ }^{8}$.

\section{MATERIAL Y MÉTODOS}

Una muestra se hizo de 6 placas de acrílico con dimensiones iguales $(2.2 \mathrm{~cm} \times 4.5 \mathrm{~cm} \times 1 \mathrm{~mm})$ a una película radiográfica con seis orificios de $5 \mathrm{~mm}$ de diámetro cada uno, cada placa se identificó con un número confeccionado de papel aluminio colocado en la parte izquierda superior, cada cemento fue mezclado de acuerdo con las especificaciones de cada fabricante, el primero de la izquierda se colocó Proroot de la Densply, segundo con MTA White de la Angelus, tercero Biodentine de la Septodont, en la segunda fila se colocó desde la izquierda el cemento Portland solo en el primer orificio, en el segundo cemento Portland con $20 \%$ de sulfato de bario y en el tercer orificio el cemento Portland con $20 \%$ de iodoformo, $1 \mathrm{~g}$ cemento por $0.37 \mathrm{ml}$ de agua destilada, en cada una de las mezclas se colocó una bolita de algodón humedecida con el fin de que ayude a fraguar de mejor manera, después se procedió a usar equipo de rayos $\mathrm{x}$ con el uso de radiovisiografo Fona y su radiopacidad determinada en densidad radiográfica con un programa software Image Tool v.3.00 se analizó la densidad radiográfica y después con análisis estadístico ANOVA.

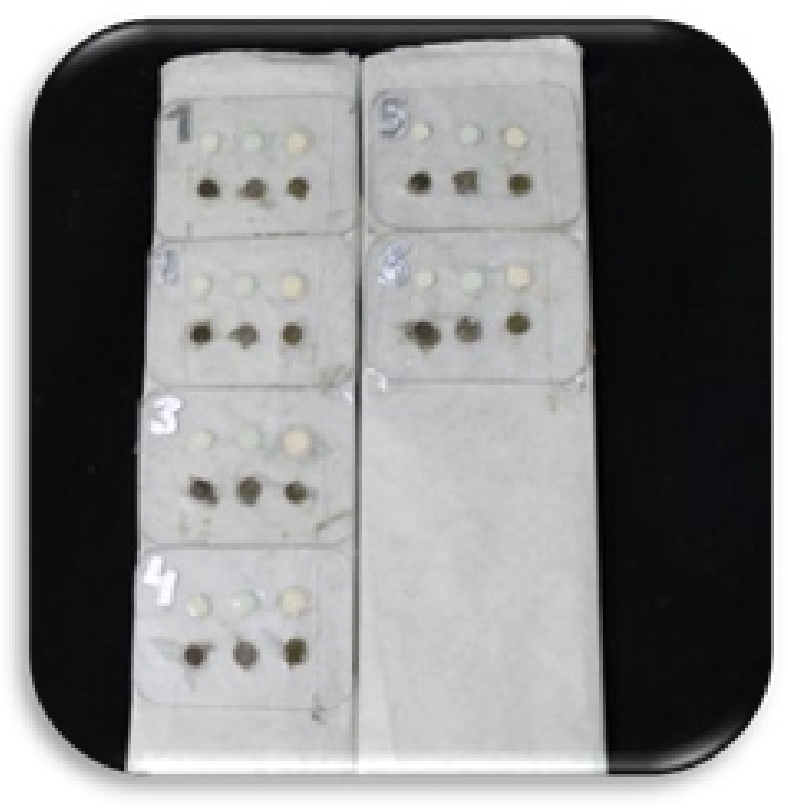

Figura 1 - Muestras. 

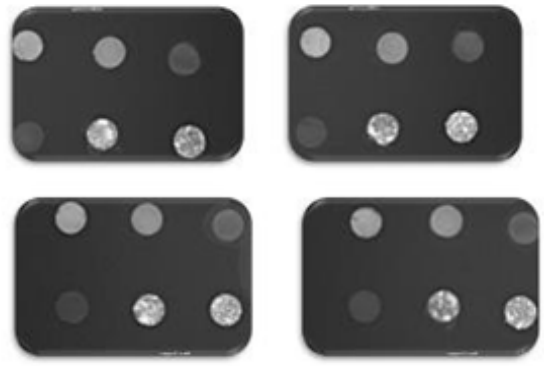

Figura 2 - Placas radiografiadas.

\section{RESULTADOS}

\begin{tabular}{|c|c|c|c|}
\hline AMOSTRA & MÉDIA & $\begin{array}{l}\text { DESVIO- } \\
\text { PADRÃO }\end{array}$ & $P$ \\
\hline PROROOT & 156.67 & 15.54 & \multirow{6}{*}{$<0.001$} \\
\hline MTAANGELUS & 145.00 & 3.16 & \\
\hline BIODENTINE* & 94.67 & 7.45 & \\
\hline PORTLAND* & 74.83 & 6.83 & \\
\hline PORTLAND+SB & 168.67 & 12.74 & \\
\hline PORTLAND+IODOFÓRMIO & 169.83 & 11.72 & \\
\hline
\end{tabular}

Figura 3 - Radiopacidad de muestras.

Análisis de variancia (ANOVA) a un criterio $(\mathrm{p}<0.05)$; *Pós-teste Tukey $(\mathrm{p}<0.05)$.

Houve diferencia estadística significativa entre los productos utilizados (ANOVA $\mathrm{p}<0.05$ ), siendo los grupos con mayores diferencias Biodentine e Portland (Tukey $\mathrm{p}<0.05$ ).

Portland + Iodofórmio e Portland + SB fueron los que presentaron mayor radiopacidad.

Portland fue el que presento menor radiopacidad.

En lo que se refiere a los MTA de casa comerciales Proroot presentó mayor radiopacidad.

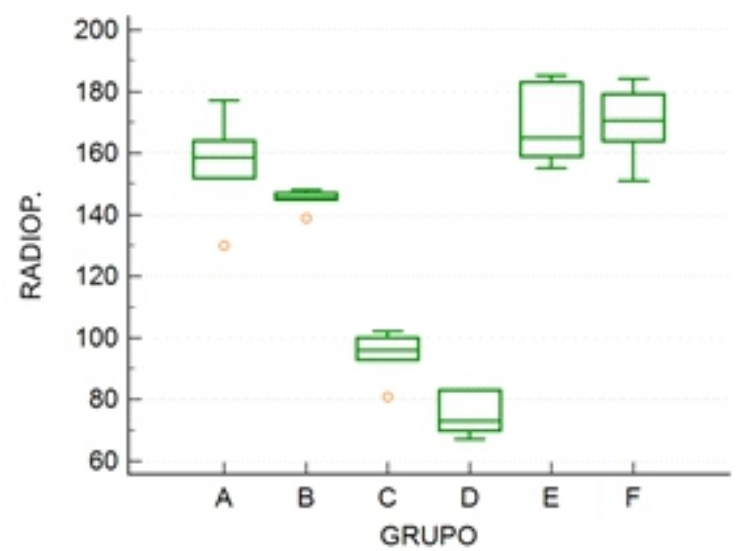

Figura 4 - Gráfico de comparaçiones múltiplas (A=PROROOT; $\mathrm{B}=$ MTA ANGELUS; $\mathrm{C}=\mathrm{BIODENTINE} ; \mathrm{D}=\mathrm{PORTLAND}$; $\mathrm{E}=$ PORTLAND+SB; F= PORTLAND+BIOD).

\section{DISCUSIÓN}

Si bien es cierto se han realizado muchos estudios sobre la radiopacidad, estos solo se han hecho solo con MTA de marcas comerciales o solo con cemento Portland añadido sustancias radiopacificadoras, este estudio trata de analizar ambos parámetros.

En lo que se trata de cemento Portland, por sí solo no posee radiopacidad al no tener ningún componente que le confiera tal propiedad, al comparar resultados de estudios en los que se le adiciona al cemento Portland con diferentes sustancias tales como: sulfato de bario y yodoformo ${ }^{7}$ se puede apreciar resultados similares es decir alta radiopacidad con dichas combinaciones. El yodoformo es un polvo de color amarillento, poco soluble en agua, soluble en alcohol y éter, con un alto porcentaje de Yodo (97\%), que presenta potencial bactericida dándole una característica importante ${ }^{8}$, mientras que el sulfato de bario es usado como medio de contraste en exámenes gastrointestinales ${ }^{7}$.

Al ver la radiopacidad de las marcas comerciales de MTA al igual que estudios realizados por el Proroot es el que mayor resultado presentó al igual que este estudio.

El cemento Portland y el Biodentine son los que menor radiopacidad $^{10}$.

Tanto el cemento Portland como el MTA tienen varias similitudes en su composición teniendo en cuenta que este último es un derivado del primero, pero el óxido de bismuto es el que da la propiedad de radiopacidad y esta presente en los MTA Proroot y Angelus, en cambio en el Biodentine está presente el dióxido de zirconio el cual le da radiopacidad".

El Biodentine es un nuevo material basado en silicato de calcio, creado por el grupo de investigadores de Septodont, para ser utilizado como un sustituto de la dentina dañada? .

\section{CONCLUSIÓN}

El cemento Portland fue la base del MTA, al cual se le adicionaron y se quitaron ciertos elementos en su composición para que sea biocompatible con el organismo y obtener de este modo un material usado ampliamente en odontología para sellar las vías de interacción entre los conductos radiculares principales y secundarios con los tejidos perirradiculares.

La radiopacidad es una propiedad que permite al MTA diferenciarlo en una radiografía de las estructuras limitantes, siendo Proroot el más radiopaco de todos los MTA utilizados en esto estudio, también se podría decir que los componentes adicionados al cemento portland como el sulfato de bario y el yodoformo poseen una radiopacidad bastante significativa. 


\section{REFERENCIAS}

1. Obando-Pereda GA. Análisis de la composición química, capacidad de sellado apical y propiedades antimicrobianas del MTA y del cemento Portland. Endodoncia. 2009;27(3):111-20.

2. Chegüe Vargas N, Juárez Broon N, Martínez Calixto L, Manzano Chaidez J, Moreno Castillo E, Cervantes Amador F. Biocompatibilidad de MTA-Angelus@y cemento Portland blanco aditivado con cloruro de calcio y clorhexidina en tejido subcutáneo de ratas. Endodoncia. 2010;28(1):7-11.

3. Martins I. Propriedades físico-químicas do MTA versus cimento de Portland [dissertation] Coimbra: Universidade de Coimbra; 2012.

4. Borges AH, Guedes OA, Volpato LER, Siebert Filho G, Borba AM, Zina O, et al. Physicochemical properties of MTA and Portland cement after addition of aloe vera. Iran Endod J. 2017;12(3):312-7.

5. Mestieri L, Tanomaru-Filho M, Gomes-Cornélio A, Salles L, Bernardi M, Guerreiro-Tanomaru J. Radiopacity and cytotoxicity of Portland cement associated with niobium oxide micro and nanoparticles. J Appl Oral Sci. 2014;22(6):554-9.

6. Aznar FDC, Bueno CE, Nishiyama CK, Martin AS. Radiopacidade de sete cimentos endodônticos avaliada através de radiografia digital. RGO. 2010;58(2):181-4.

7. Rosas Solís PA, Morín Alcázar NL, Quintanar Zúñiga RE, Llamosas Hernáncez EF. Radiopacidad del cemento Portland adicionado con diferentes sustancias radiopacas para su uso en perforaciones radiculares. Endod Act. 2013;8(1):22-9.

8. Marciano M, Costa R, Camilleri J, Mondelli R, Guimarães B, Duarte M. Assessment of color stability of white mineral trioxide aggregate angelus and bismuth oxide in contact with tooth structure. J Endod. 2014;40(8):1235-40.

9. Kaup M, Schäfer E, Dammaschke T. An in vitro study of different material properties of Biodentine compared to ProRoot MTA. Head Face Med. 2015;11(1):16.

10. Gil AMC, Herrera LH. Generalidades del agregado de trióxido mineral (mta) y su aplicación en odontología: revisión de la literatura. Acta Odontol Venezolana. 2007;45(3):8. 